\title{
Weather Measurements with Multi-mission Phased Array Radar Challenges to meet Requirements
}

\author{
D.S. Zrnic \\ NOAA/OAR National Severe Storms Laboratory, Norman, Oklahoma
}

\begin{abstract}
This paper identifies the most challenging requirements for weather observations that a future Polarimetric Phased Array Radar (PPAR) should meet. These are routed in the current capabilities of the US national network of Doppler Weather Radars (WSR-88D). Biases in polarimetric variables are related to the PPAR designs and polarimetric mode of operations. A planar-array PPAR being used for resolving some technical challenges is described.
\end{abstract}

Index Terms - Weather radar, phased array radar, polarimetric measurements, errors in polarimetric variables.

\section{INTRODUCTION}

Exploration of agile beam phased array radar (PAR) technology for weather application is in its infancy and is the prime motivator for this presentation. The PAR is being considered for Multi-mission applications (MPAR) to serve aviation and weather needs as well or better than the current mission specific radars [1]. Potent novelty of rapid shift in beam position, quick change in operating parameters, and adaptive beam forming are the advantages of the PAR.

Implementation of polarimetric measurements imposes strict requirements on the antenna's performance to attain quantitative precipitation measurements and to classify precipitation types. These requirements are hard to meet with the Polarimetric PAR (PPAR) because such radar can produce thousands of beams with varying gain and beamwidth, each of which requires precise calibration. Most critical is to have a negligible cross-polar beam coaxial with the copolar beam [2]. Currently two antenna geometries are under consideration, a four face planar antenna array and a cylindrical antenna array.

\section{REQUIREMENTS FOR WEATHER SURVEILLANCE}

Some of the original challenging requirements are listed in [3] and the ones for polarimetric measurements came later. The WSR-88D has exceeded many of the requirements thus the PPAR should meet or exceed its current ones. Those listed in the Table 1 are most pertinent. The beamwidth and far sidelobe specifications can be achieved at considerable cost but with existing knowhow. Recent solid state amplifier can produce peak power several tens $\mathrm{W}$ so the PPAR sensitivity can match the requirement even without pulse compression. The pulse compression introduces additional complications. For covering range down to $2 \mathrm{~km}$ a short pulse at a different frequency is needed; this would consume a portion of allocated bandwidth. Other issues are the range sidelobes of compressed pulse, matching of the transition in range between the short and long pulse, and a bit longer dwell time.

The currently allocated bandwidth of $14.5 \mathrm{MHz}$ (Table I) might be increased for the MPAR because the total number of radars would be reduced by about 200. Consequential increase in spatial separation could relax the requirement for frequency separation. Moreover, by fulfilling the ARSR mission but at $\mathrm{S}$ band, the $\mathrm{L}$ band allocated to the ARSR would be freed and possibly traded for an increase at $\mathrm{S}$ band? Nonetheless, the bandwidth will remain the most precious constraining commodity. Rather than expanding it for pulse compression purposes a wiser use would be for multiple beam and multiple face operations [4].

The required polarimetric variables are: differential reflectivity $Z_{\mathrm{DR}}=10 \log \left(P_{\mathrm{h}} / P_{\mathrm{v}}\right)$; copolar correlation coefficient $\rho_{h v}=\left|\operatorname{cor}\left(V_{h h}^{*} V_{v v}\right)\right|$, and differential phase $\Phi_{\mathrm{DP}}=\arg \left(\rho_{\mathrm{hv}}\right)$. In

TABLE I

SOME WSR-88D SPECIFICATIONS

\begin{tabular}{|c|c|}
\hline $\begin{array}{l}\text { Beamwidth } \\
\text { One way far sidelobes' level } \\
\text { Sensitivity: } 1 \mu \mathrm{s} \text { pulse yields SNR }>0 \text { if the volume is filled with } \\
\text { scatterers } \\
\text { Transmission bandwidth at }-40 \mathrm{dBc} \text { [ appendix B] }\end{array}$ & $\begin{array}{l}1 \mathrm{o} \\
\text { Lower than }-50 \mathrm{~dB} \\
\text { For } \mathrm{Z}=-11 \mathrm{dBZ} \text { at } \mathrm{r}=50 \mathrm{~km} \\
14.5 \mathrm{MHz}\end{array}$ \\
\hline $\begin{array}{l}\text { Unambiguous range for relfectivity and polarimetric variables } \\
\text { Minimum range }\end{array}$ & $\begin{array}{l}466 \mathrm{~km} \\
2 \mathrm{~km}\end{array}$ \\
\hline $\begin{array}{ll}\text { Clutter canceling: } & \begin{array}{l}\text { for Doppler processing } \\
\text { for reflecitivyty and polarimetric variables }\end{array} \\
\end{array}$ & $\begin{array}{l}50 \mathrm{~dB} \\
30 \mathrm{~dB}\end{array}$ \\
\hline 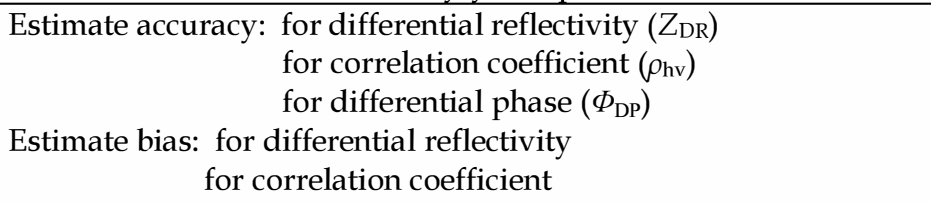 & $\begin{array}{l}\leq 0.3 \mathrm{~dB} ; \text { at } S N R>20 \mathrm{~dB} ; \sigma_{\mathrm{v}}=2 \mathrm{~m} \mathrm{~s}^{-1} \\
\leq 0.006 ; \text { at } S N R>20 \mathrm{~dB} ; \sigma_{\mathrm{v}}=2 \mathrm{~m} \mathrm{~s}^{-1} \\
\leq 2.5^{\circ} ; \text { at } S N R>20 \mathrm{~dB} ; \sigma_{\mathrm{v}}=2 \mathrm{~m} \mathrm{~s}^{-1} \\
\leq|0.1| \mathrm{dB} \\
\leq|0.01|\end{array}$ \\
\hline
\end{tabular}


these expressions $P_{\mathrm{h}}$ is the power corresponding to the returns at horizontal polarization, and $P_{\mathrm{v}}$ at vertical. The $V_{\mathrm{hh}}$ is voltage corresponding to transmitted field at horizontal polarization and received at horizontal polarization; similarly $V_{\mathrm{vv}}$ refers to vertical polarization.

At elevations below about $2.5^{\circ}$ the polarimetric variables are obtained at a long PRT (about $3 \mathrm{~ms}$ ) and thus are free of range overlaid signals because the beam at the lowest $\left(0.5^{\circ}\right)$ elevation is above storm tops. At such long PRT Doppler ambiguities encountered in severe storms can't be resolved, hence a separate scan at about three to four times larger PRT is used.

Clutter recognition relies on the phase of the autocorrelation spectral density at lag one [5] and is followed by an adaptive spectral filter. To achieve the desired rejection sufficient dwell time is needed. On the PPAR one can apply spatial clutter filtering and beam shaping [6]. This combined with spectral filters might provide better overall clutter removal and at a shorter dwell times. Note that on the PPAR there is no beam smearing allowing better removal of clutter while preserving more of the weather signal. Optimum multifaceted clutter filtering on the PPAR is definitely a worthy, albeit no urgent subject for further research.

\section{POLARIMETRIC MODES}

In its simplest form the relation between the incident field $\mathbf{E}^{\mathbf{i}}$ and scattered $\mathbf{E}^{\mathbf{s}}$ can be expressed as

$$
\left[\begin{array}{c}
E_{\theta}^{s} \\
E_{\phi}^{s}
\end{array}\right]=\frac{e^{-j k r}}{r}\left[\begin{array}{cc}
s_{\mathrm{hh}} e^{j \Phi_{D P}} & 0 \\
0 & s_{\mathrm{vv}}
\end{array}\right]\left[\begin{array}{c}
E_{\theta}^{l} \\
E_{\phi}^{l}
\end{array}\right]
$$

where the $s_{\mathrm{hh}}$ and $s_{\mathrm{vv}}$ are the backscatter matrix terms, $r$ is range and $k$ wavenumber. The propagation medium consist of precipitation that is horizontally aligned hence the off diagonal terms of (1) are zero. Attenuation is negligible, but the phase difference $\Phi_{\mathrm{DP}}$ due to the system, propagation and backscattering is important. For the dish antenna the intended horizontally polarized field $E_{h}=E_{\phi}^{l}$ and vertically polarized field $E_{v}=E_{\theta}^{l}$ are invariant with respect to beam position. The horizontal is parallel to the earth surface and the vertical is in the plane of propagation.

Two polarimetric modes are considered for the PPAR: The alternate (AHV) in which successive transmitted pulses alter polarization between horizontal and vertical and on reception both polarizations are captured. The simultaneous (SHV) in which an elliptical polarization (with axis at $45^{\circ}$ or $-45^{\circ}$ ) is transmitted but linear horizontal and vertical polarized returns are processed.

Because (1) is a faithful representation of physics, obtaining polarimetric variables in the simultaneous (SHV) mode is possible. The AHV has the disadvantage that in the surveillance scan ( $3 \mathrm{~ms}$ PRT) errors of polarimetric variables are excessive. The example in Fig. 1 shows standard error of $\Phi_{\mathrm{DP}}$. Beyond the spectrum width of $2 \mathrm{~m} \mathrm{~s}^{-1}$ it is unacceptable as it would occur more than $50 \%$ of the time in most storms (median is $2 \mathrm{~m} \mathrm{~s}^{-1}$; in squall lines it is $4 \mathrm{~m} \mathrm{~s}^{-1}$
[7]). The other polarimetric variables exhibit similarly devastating errors in the AHV long range mode disqualifying it from use on the PPAR in the long range mode. The other drawbacks of the AHV are: coupling between the Doppler velocity and $\Phi_{\mathrm{DP}}$, indirect measurement of $\rho_{h v}$, and reduction by a factor of two in the placements of filter notches for clutter rejection. Because the SHV mode is prone to bias errors induced by the antenna the challenge is
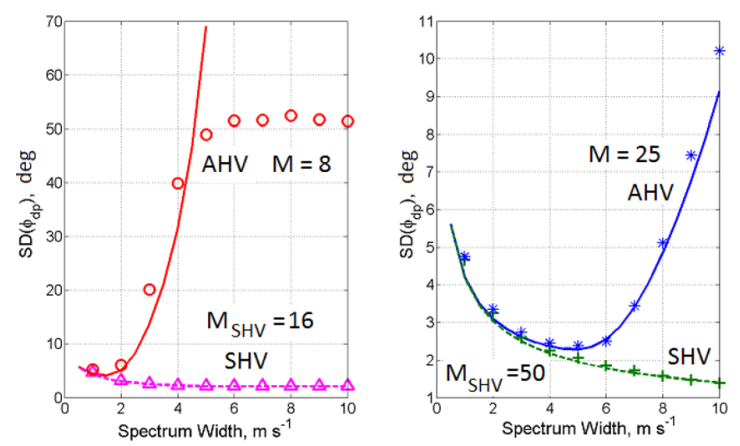

Fig. 1. Standard deviations of $\Phi_{\mathrm{DP}}$ for the AHV (solid curves) and SHV (dash curves) modes. The results are theoretical; symbols represent simulations. On the left panel the PRF $=320 \mathrm{~Hz}$ on the right it is $1000 \mathrm{~Hz}, \mathrm{SNR}>20 \mathrm{~dB}$. In either case $\rho_{h v}=0.98$.

to develop a variant less affected by these errors. Promising approaches are in [8] and will be presented.

\section{CAUSES OF BIAS}

Various causes of bias are discussed and their relative contributions are compared in this section.

\section{A. Geometrically induced coupling}

The conceptual drawing in Fig. 2 helps highlight one issue in making polarimetric measurements with the planar PAR. The $h$ and $v$ axis define the plane of polarization and the vector incident fields to the left are at the beam center

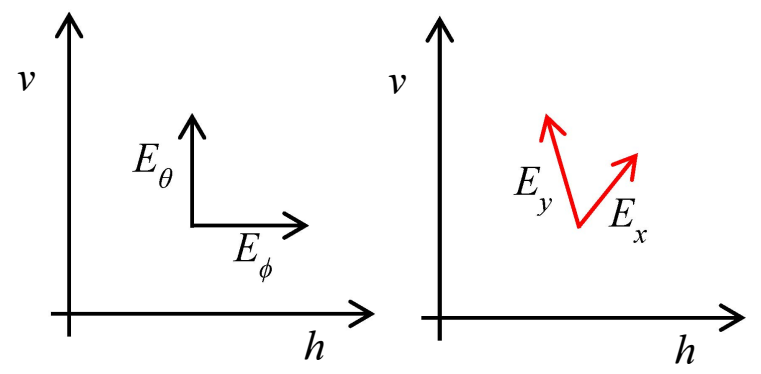

Fig. 2. Electric Fields in the center of the beam. Black vectors do not induce geometric bias, the red ones do.

of a parabolic dish. Fields $E_{x}$ and $E_{y}$ to the right are at beam center of a planar PPAR pointing out of the principal planes. The $E_{x}$ is caused by energizing the port $x$ which would produce the $\mathrm{H}$ polarization in the principal plane. The $E_{y}$ is from the energized port $y$. Out of the principal planes these 
ports produce fields that may not be orthogonal, equal, or collinear with the $h$ or $v$ axes, i.e., the unit vectors $\hat{x}, \hat{y}$ form a non-orthonormal basis. This coupling is geometrically induced and dependent on the type or radiating elements. The polarimetric measurements need to be made in the $\hat{h}, \hat{v}$ basis for quantitative interpretation of precipitation. In theory it is possible to convert the polarimetric measurements made in the $\hat{x}, \hat{y}$ basis to the $\hat{h}, \hat{v}$ basis [9]-[10]. Conversion requires knowledge of the orientation of the transmitted field at every pointing direction. This, in practice is a very hard to solve problem.

To avoid the geometrically induced coupling a collinear magnetic and electric dipoles have been proposed [11]. Such configurations are three dimensional and therefore much costlier than typical two dimensional patch radiators. No functional PPAR antenna exhibiting these coaxial dipoles has been tested. Cylindrical PPAR [12] that scans by commutation in azimuth also has no geometrically induced bias and is under development [13]-[14].

\section{B. Cross coupling through the radiation pattern}

The unavoidable bias in PPAR is due to cross coupling through the radiation patterns. Let the copolar voltage patterns be $F_{\mathrm{hh}}$ and $F_{\mathrm{vv}}$, and the cross-polar be $F_{\mathrm{vh}}$ and $F_{\mathrm{hv}}$. These complex quantities can be expressed as $F_{\mathrm{ij}}(\theta, \phi)=\sqrt{g_{\mathrm{ij}}} f_{\mathrm{ij}}(\theta, \phi)$ and include gain $g_{\mathrm{ij}}$. A voltage return from a single scatterer illuminated by the pattern is

$$
\left[\begin{array}{l}
\delta V_{\mathrm{h}} \\
\delta V_{\mathrm{v}}
\end{array}\right]=\left[\begin{array}{ll}
F_{\mathrm{hh}} & F_{\mathrm{vh}} \\
F_{\mathrm{hv}} & F_{\mathrm{vv}}
\end{array}\right]\left[\begin{array}{cc}
s_{\mathrm{hh}} e^{j \Phi_{\mathrm{pp}}} & 0 \\
0 & s_{\mathrm{vv}}
\end{array}\right]\left[\begin{array}{ll}
F_{\mathrm{hh}} & F_{\mathrm{hv}} \\
F_{\mathrm{vh}} & F_{\mathrm{vv}}
\end{array}\right]\left[\begin{array}{c}
1 \\
e^{j \beta}
\end{array}\right],
$$

where the differential phase on transmission is $\beta$. As written the equation applies to the SHV mode. To represent the AHV mode alternate the leftmost vector between $[1,0]^{\mathrm{T}}$ and $\left[0, e^{j \beta}\right]^{\mathrm{T}}$. Integrals over the main beam of power contribution by scatterers are introduced in (1) to reveal the biases due to the $F_{\mathrm{vh}}$ and $F_{\mathrm{hv}}$. The biases in $Z_{\mathrm{DR}}$ and $\rho_{\mathrm{hv}}$ depend on $\beta, \Phi_{\mathrm{DP}}$, and the cross- polar complex pattern. The biases differ significantly in the AHV and SHV mode. Intuitive explanation is in Fig. 3.

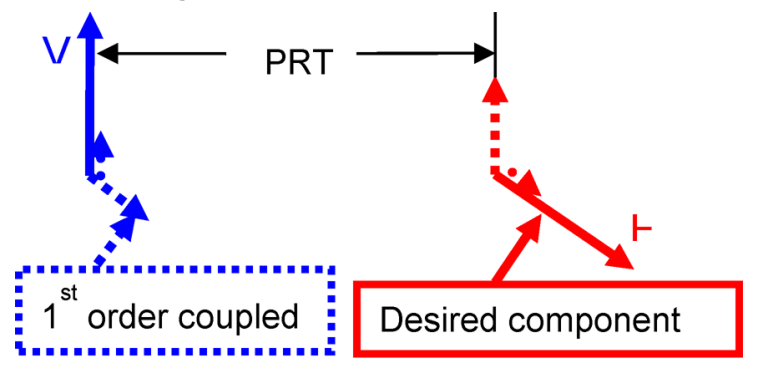

Fig. 3. Sketch of the V polarized field followed by the $H$ polarized one in the SHV mode.
Note that in the AHV mode the first order coupled component (dashed blue $\mathrm{H}$ vector) is in the orthogonal channel which is dormant on reception leaving only the second order coupled terms; these are caused by double coupling, once from $\mathrm{V}$ to $\mathrm{H}$ on transmission then back to $\mathrm{V}$ on reception. Thus the largest bias term contains $F_{v h}^{2}$ and it is

$$
R_{A H V}=2 \int_{\Omega} F_{\mathrm{hh}}^{2} F_{\mathrm{vh}}^{2} .
$$

In the SHV mode, coupled are the first order terms hence the largest bias term contains $F_{\mathrm{vh}}$ to the first power and is

$$
R_{S H V}=4 \int_{\Omega} F_{\mathrm{hh}}^{3} F_{\mathrm{vh}} .
$$

Defining the integrated cross-polar power ratio $\Lambda$ as

$$
\Lambda=\int_{\Omega} F_{\mathrm{vh}}^{2} / \int_{\Omega} F_{\mathrm{hh}}^{2}
$$

it follows that if the cross-polar pattern coincides in shape with the copolar (i.e., $F_{\mathrm{vh}}=c F_{\mathrm{hh}}$ ), the $Z_{\mathrm{DR}}$ bias would be same in SHV and AHV mode provided

$$
\Lambda_{\mathrm{SHV}}(\mathrm{dB})=2 \Lambda_{A H V}(\mathrm{~dB})-6 .
$$

Clearly the SHV mode requires twice (in $\mathrm{dB}$ ) the isolation of the AHV mode to achieve the same bias control.

Note that this discourse is equally applicable to EM coupling through the circuits and other component on the system. This is because such coupling is indistinguishable from coupling in which $F_{\mathrm{vh}}=c F_{\mathrm{hh}}$.

In Fig. 4 is the maximum positive $Z_{\mathrm{DR}}$ bias parameterized according to $\beta$ and $\gamma$ which is the phase difference between $F_{\mathrm{vh}}$ and $F_{\mathrm{hh}}$. The parameter $W=\left|\int_{\Omega} F_{\mathrm{hh}}^{3} F_{\mathrm{vh}} / \int_{\Omega} F_{\mathrm{hh}}^{4}\right|$.

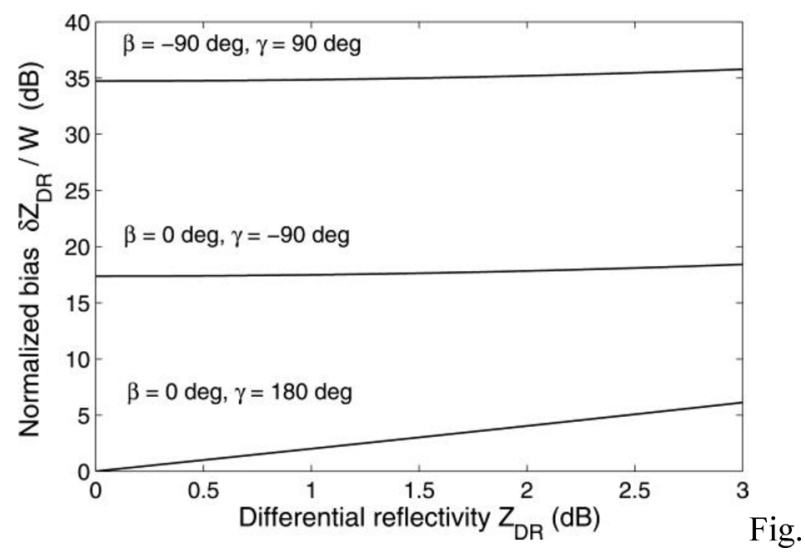

Fig. 4. Envelopes of the maximum positive $Z_{\mathrm{DR}}$ bias normalized by $W$ (linear scale), for $\rho_{\mathrm{hv}}=1$, and the indicated values of the phases (from [2]).

The worst case bias is if transmitted polarization is circu$\operatorname{lar}\left(\beta=-90^{\circ}\right)$ and the smallest is if the polarization is slant linear and $\gamma=180^{\circ}$. In practice it would be extremely hard to control $\gamma$ as it is a composite from all coupling effects. Adjusting $\beta$ to zero should be possible.

To overcome the large $Z_{\mathrm{DR}}$ bias in the SHV mode, pulse to pulse phase coding has been proposed [8]. It is at a cost 
of increased estimate variance and increased bias of $\rho_{\mathrm{hv}}$.

\section{Mismatch of gains and beam cross sections}

The gains and beam cross sections enter as product terms in the radar equation, hence the bias in $Z_{\mathrm{DR}}$ is

$$
\text { Bias }=10 \log \left(\frac{g_{h}^{2} \theta_{h, L}^{2}}{g_{v}^{2} \theta_{v, L}^{2}}\right) .
$$

Assumption is that the beams' cross sections are circular Gaussian and the $\theta_{\mathrm{L}} \mathrm{s}$ are widths measured at a level $L$ below the peak of the main lobe. It the $\mathrm{H}$ pattern width is measured at the $L$ level and the $\mathrm{V}$ pattern is either wider (has the same width at a higher level $-L+\Delta$ ) or narrower (has the same width at $-L-\Delta)$ the bias from (8) becomes $\pm 10 \Delta / L \log (e)$. Take $L=20$ below the peak. Then it follows that $\Delta$ should be $0.5 \mathrm{~dB}$ to keep the bias below $0.1 \mathrm{~dB}$.

\section{CALIBRATION}

Maintaining calibration of a PPAR to weather radar standards but in the field has never been done. Herein I list the important parameters that need to be calibrated. Accounting for the geometrically induced bias is specific to planar arrays with identical elements for the $\mathrm{H}$ and $\mathrm{V}$ polarization. It has not been demonstrated and NSSL is involved in studying the issue (section V).

The variables that need calibration are $Z$ at each pointing direction to within $1 \mathrm{~dB}$. This continues to be a problem on the WSR-88D and will be much harder on the PPAR because it requires determination of the change in gain and beam cross section for every pointing direction.

Calibrating $Z_{\mathrm{DR}}$ might be as hard if not harder than calibrating $Z$. Monitoring and frequent adjustments will be needed to maintain it stable during operation. On the WSR$88 \mathrm{D}$ the relative $Z_{\mathrm{DR}}$ bias offset is maintained via calibration of the two receiver chains at $5 \mathrm{~min}$ intervals between volume scans. Similar procedure might be needed but with accounting for the variations of difference in transmitted $\mathrm{H}$ and $\mathrm{V}$ powers. On most conventional radars, excluding the WSR-88D, verification of $Z_{\mathrm{DR}}$ calibration is achieved by pointing the antenna vertically. In that direction the expected value of $Z_{\mathrm{DR}}$ is zero and the system should be adjusted accordingly. This however will not be possible on the PPAR. Occasional verification could be made in light rain, drizzle, reflection of Brag scatterers, or aggregates.

The variation of the system differential phase $\Phi_{\mathrm{DPsys}}$ needs to be constrained to few tens of degrees. This should be relatively straight forward because by design the phases of all elements at broad side are aligned (known) for both polarizations. Therefore the difference of the phase in the $\mathrm{H}$ channel and $\mathrm{V}$ channel would be automatically available.

\section{DEVELOPMENTS OF VARIOUS CONCEPTS}

Several aspects of PPAR are being explored by the University of Oklahoma (OU) and NSSL. These include radiating elements, pulse compression, scanning strategies, signal processing, clutter canceling and more. A substantial effort has been expanded by the OU to develop a cylindrical PPAR [13]-[14].

NSSL has obtained from the Lincoln Laboratory (LL) a $2 \times 5$ panel PPAR (Fig.5) for testing. Its antenna can be positioned in azimuth and elevation and can have the broadside beam point vertically. It has $10 \mathrm{~cm}$ wavelength and beamwidth of about 3 and 7 degrees. Sensitivity is such that a $\mathrm{Z}$ of $0 \mathrm{dBZ}$ at $2 \mathrm{~km}$ range produces a $\mathrm{SNR}=0 \mathrm{~dB}$ if the pulse width is $1.6 \mu \mathrm{s}$. The radar can operate in both SHV and AHV mode.

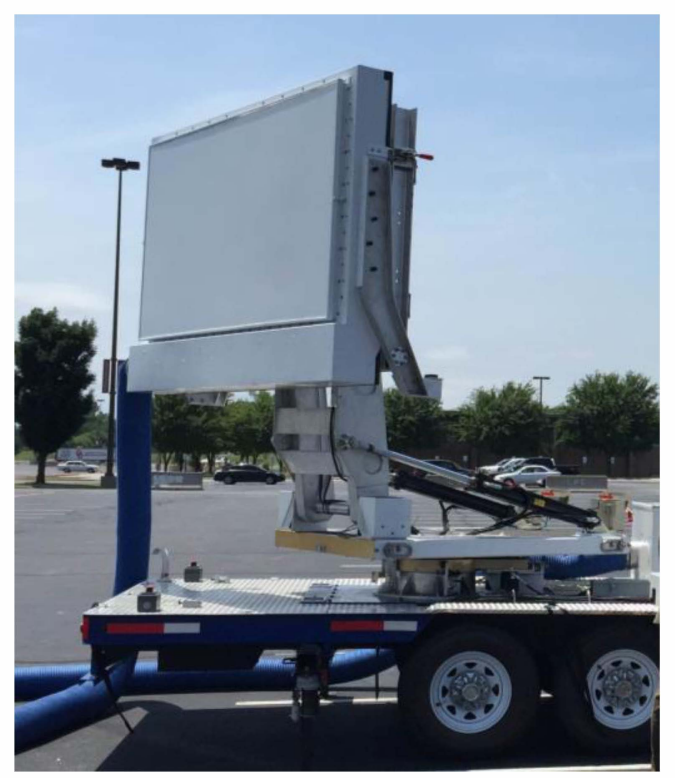

Fig. 5 The PPAR in Norman OK.

The normalized $\left|F_{h h}^{2}\right|$ and normalized $\left|F_{v h}^{2}\right|$ receiving patterns are presented in Fig.6. The pointing direction for these pattern measurements was set at elevation of $14^{\circ}$ and azimuth of $28^{\circ}$. Because this is a near field measurement the cross-polar pattern contains contributions from all coupling sourced (transceivers, microwave circuits, geometry, imperfection of the probe, etc.). Therefore its $\sim-25 \mathrm{~dB}$ peak below the co-polar peak is the worst one can expect. Still, it is a long ways from the desired $\sim-45 \mathrm{~dB}$ that is needed to make $Z_{\mathrm{DR}}$ bias smaller than $0.1 \mathrm{~dB}$ in the SHV mode. A conspicuous peak in the copolar pattern at broad side is caused by direct coupling in the transceivers. We plan to test various methods to reduce the biases and obtain accurate polarimetric variables.

The definite advantage of this system is that it can provide measurement at vertical and near vertical incidence. Thus theoretical prediction for correction of the variation of polarimetric variables with zenith angle can be compared to the measured ones. These tests can be done by interleaving the AHV and SHV for comparison of biases. At near vertical incidence we expect that two beams axially symmetric with respect to broad side would exhibit same biases in $Z_{\mathrm{DR}}$. Such and similar symmetric arrangements should establish errors, and limitations of the system. Because the 
radiating elements are patch antennas and the array is planar, the system is the most difficult to calibrate.
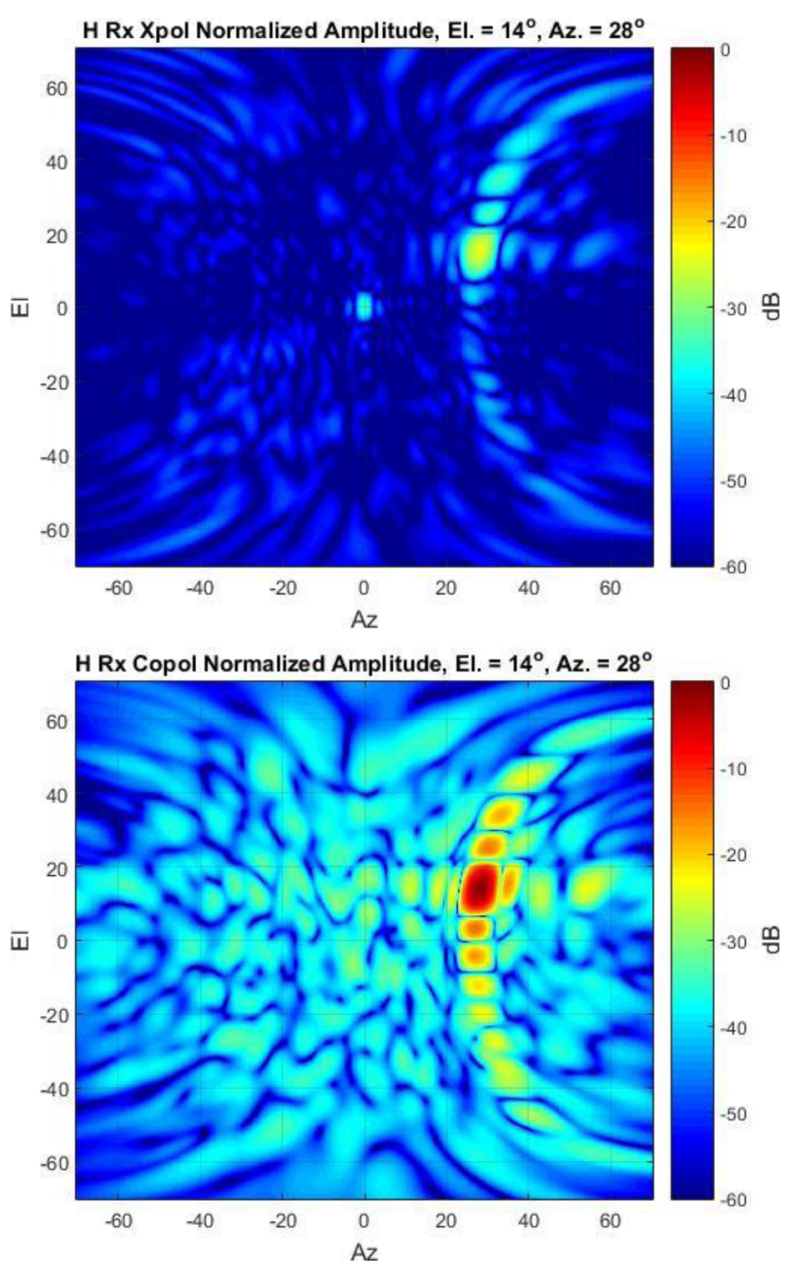

Fig. 6.Top) Copolar pattern. Bottom) Cross polar pattern.

\section{CONCLUSION}

To achieve polarimetric measurements without overlaid echoes in range to at least $460 \mathrm{~km}$ is a very hard requirement for the PPAR. In principle it can be met with the SHV polarimetric mode. But to mitigate contamination through coupling of the $\mathrm{H}$ and $\mathrm{V}$ signals in this mode requires twice (in $\mathrm{dB}$ ) the isolation compared to the needed isolation in the AHV mode. Some ways to lessen the effect of coupling on the polarimetric variables using modifications of the SHV mode will be presented.

Satisfactory calibration of $Z$ and $Z_{\mathrm{DR}}$ has never been demonstrated in the field. On an ideal cylindrical PPAR this issue is easier to handle than on ideal planar PPAR. This is because the gain and resolution volume size change only with elevation on the cylindrical PPAR but change with both elevation and azimuth on the planar PPAR.

Planned calibration for a planar PPAR is suggested and results will be shown.

\section{REFERENCES}

[1] M.E. Weber, J.Y.N. Cho, J.S. Herd, J.M. Flavin, W.E. Benner, and G.S. Torok, "The next-generation multimission U.S. surveillance radar network," Bulletin of the Amer. Meteor. Soc., vol. 88, no. 11, 2007.

[2] D.S. Zrnić, R. J. Doviak, G. Zhang, and A. Ryzhkov, "Bias in differential reflectivity due to cross-coupling through the radiation patterns of polarimetric weather radars," J. Atmos. Oceanic Technol., vol. 27, no. 10, 2010.

[3] NTR, 1991: NEXRAD Technical Requirements, NEXRAD Joint System Program Office (SPO1), Silver Spring, MD.

[4] D.S. Zrnić, V.M. Melnikov, R. J. Doviak, and R. Palmer, "Scanning and Waveform for the Multifunction Phased Array Radar to satisfy Aviation and Meteorological Needs" IEEE Geos. Remote Sens. Let., vol. 12, 2014.

[5] A.D. Warde and S. M. Torres, "Automatic detection and removal of ground clutter contamination on weather radars," 34th Conference on Radar Meteorology, AMS, Williamsburg, VA, USA, 2009.

[6] C. D. Curtis and M. Yeary, "Adaptive beamforming to mitigate ground clutter on the National Weather Radar Testbed phased array radar." IEEE Trans. Microw. Theory Techn., submitted.

[7] M. Fang, R.J. Doviak, and V. Melnikov, " Spectrum width measured by WSR-88D: error sources and statistics of various weather phenomena" Jour. Atmos. Oceanic Technol., vol. 21, 2004.

[8] D.S. Zrnic, R.J. Doviak, V.M. Melnikov, and I. Ivic, "Signal design to suppress coupling in the SHV polarimetric mode," Jour. Atmos. Oceanic Technol., vol 31, No. 5, 2014.

[9] G. Zhang, R. J. Doviak, D. S. Zrnic, G. Crain, D. Staiman, and Y. Al-Rashid, "Phased array polarimetry for weather sensing: a theoretical formulation for bias corrections," IEEE Trans. Geoscience and Remote Sensing, vol. 47, no. 11, 2009.

[10] D.S. Zrnic, G. Zhang, and R.J. Doviak, "Bias correction and Doppler measurement for polarimetric phased-array radar" ," IEEE Trans. Geoscience and Remote Sensing, vol. 49, no. 2, 2011.

[11] G.E. Crain, and D. Staiman, "Polarization selection for phased array weather radar" IIPS for Meteorology, Oceanography, and Hydrology, 25th Int. Conf. on, New Orleans, AMS, Jan. 2009.

[12] G. Zhang, R. J. Doviak, D. S. Zrnic, R. D. Palmer, L. Lei, Y. Al-Rashid, "Polarimetric phased array radar for weather measurement: a planar or cylindrical configuration?" J. Atmos. Ocean. Tech., vol. 28, no. 1, 2011.

[13] R. Kelley,"Cylindrical Polarimetric Phased Array Radar: Hardware Design and Mobile Demonstrator" IEEE Array 2013 Conference.

[14] S. Karimkashi, "Cylindrical Polarimetric Phased Array Radar: Design and Analysis of a Frequency Scanning Antenna Array" IEEE Array 2013 Conference. 